\title{
A MULTIPARAMETER SUMMATION FORMULA FOR RIEMANN THETA FUNCTIONS
}

\author{
VYACHESLAV P. SPIRIDONOV
}

\begin{abstract}
We generalize Warnaar's elliptic extension of a Macdonald multiparameter summation formula to Riemann surfaces of arbitrary genus.
\end{abstract}

We start by a brief outline of the general theory of hypergeometric type series built from Jacobi theta functions [13, 14] (its extension to integrals [16 is not touched at all). Within this approach, univariate elliptic hypergeometric series are defined as formal series $\sum_{n} c_{n}$ for which $h(n)=c_{n+1} / c_{n}$ is an elliptic function of $n$ considered as a continuous complex variable. Normalizing $c_{0}=1$, we see that all coefficients $c_{n}$ are obtained as products of $h(k)$ or $1 / h(k)$ for different $k \in \mathbb{Z}$.

Any elliptic function of order $r+1$ can be represented as [19]:

$$
\begin{aligned}
& h(n)=z \frac{\theta_{1}\left(u_{0}+n, \ldots, u_{r}+n ; \sigma, \tau\right)}{\theta_{1}\left(v_{0}+n, \ldots, v_{r}+n ; \sigma, \tau\right)}=z \frac{\theta\left(t_{0} q^{n}, \ldots, t_{r} q^{n} ; p\right)}{\theta\left(w_{0} q^{n}, \ldots, w_{r} q^{n} ; p\right)} \\
& \theta_{1}\left(u_{0}, \ldots, u_{k} ; \sigma, \tau\right)=\prod_{i=0}^{k} \theta_{1}\left(u_{i} ; \sigma, \tau\right), \quad \theta\left(t_{0}, \ldots, t_{k} ; p\right)=\prod_{i=0}^{k} \theta\left(t_{i} ; p\right),
\end{aligned}
$$

with a free variable $z \in \mathbb{C}$, the base $p=e^{2 \pi i \tau}$ satisfying the constraint $|p|<1$ (or $\operatorname{Im}(\tau)>0)$, and arbitrary $q=e^{2 \pi i \sigma}$. The function

$$
\begin{aligned}
\theta_{1}(u ; \sigma, \tau) & =-i \sum_{k=-\infty}^{\infty}(-1)^{k} p^{(2 k+1)^{2} / 8} q^{(k+1 / 2) u} \\
& =i p^{1 / 8} q^{-u / 2}(p ; p)_{\infty} \theta\left(q^{u} ; p\right), \quad u \in \mathbb{C},
\end{aligned}
$$

is the standard Jacobi $\theta_{1}$-function. The short theta function $\theta(a ; p)$ has the form

$$
\theta(a ; p)=(a ; p)_{\infty}\left(p a^{-1} ; p\right)_{\infty}, \quad(a ; p)_{\infty}=\prod_{n=0}^{\infty}\left(1-a p^{n}\right)
$$

and obeys the properties

$$
\theta(p a ; p)=\theta\left(a^{-1} ; p\right)=-a^{-1} \theta(a ; p) .
$$

We use also the convenient elliptic numbers notation:

$$
[u ; \sigma, \tau] \equiv \theta_{1}(u ; \sigma, \tau), \quad \text { or } \quad[u] \equiv \theta_{1}(u) .
$$

The parameters $u_{i}$ and $v_{i}$ in (11) are defined modulo shifts by $\sigma^{-1}$, which will not be indicated in the formulas below. They are connected to $t_{i}$ and $w_{i}$ as

$$
t_{i}=q^{u_{i}}, \quad w_{i}=q^{v_{i}}
$$

2000 Mathematics Subject Classification. Primary 33E20; secondary 14H42.

Date: November 2004; Proceedings of the Workshop on Jack, Hall-Littlewood and Macdonald polynomials (Edinburgh, September 23-26, 2003), Contemp. Math., to appear. 
and satisfy the constraint

$$
\sum_{i=0}^{r}\left(u_{i}-v_{i}\right)=0, \quad \text { or } \quad \prod_{i=0}^{r} t_{i}=\prod_{i=0}^{r} w_{i}
$$

which guarantees double periodicity of the meromorphic function $h(n)$ :

$$
h\left(n+\sigma^{-1}\right)=h(n), \quad h\left(n+\tau \sigma^{-1}\right)=h(n) .
$$

For the entire function $[u]$ we have $[-u]=-[u]$,

$$
\left[u+\sigma^{-1}\right]=-[u], \quad\left[u+\tau \sigma^{-1}\right]=-e^{-\pi i \tau-2 \pi i \sigma u}[u],
$$

and

$$
\begin{aligned}
& {[u ; \sigma, \tau+1]=e^{\pi i / 4}[u ; \sigma, \tau],} \\
& {[u ; \sigma / \tau,-1 / \tau]=-i(-i \tau)^{1 / 2} e^{\pi i \sigma^{2} u^{2} / \tau}[u ; \sigma, \tau],}
\end{aligned}
$$

where the sign of $(-i \tau)^{1 / 2}$ is fixed from the positivity of its real part condition. The latter two transformations generate the modular $P S L(2, \mathbb{Z})$-group,

$$
\tau \rightarrow \frac{a \tau+b}{c \tau+d}, \quad \sigma \rightarrow \frac{\sigma}{c \tau+d},
$$

where $a, b, c, d \in \mathbb{Z}$ and $a d-b c=1$.

For the unilateral series $\sum_{n \in \mathbb{N}} c_{n}$, we conventionally normalize $w_{0}=q$ (or $\left.v_{0}=1\right)$. Then, the elliptic function $h(n)$ generates the single variable elliptic hypergeometric series:

$$
{ }_{r+1} E_{r}\left(\begin{array}{c}
t_{0}, \ldots, t_{r} \\
w_{1}, \ldots, w_{r}
\end{array} ; q, p ; z\right)=\sum_{n=0}^{\infty} \frac{\theta\left(t_{0}, t_{1}, \ldots, t_{r} ; p ; q\right)_{n}}{\theta\left(q, w_{1}, \ldots, w_{r} ; p ; q\right)_{n}} z^{n} .
$$

The elliptic shifted factorials are defined as

$$
\theta\left(t_{0}, \ldots, t_{k} ; p ; q\right)_{n} \equiv \prod_{m=0}^{k} \prod_{j=0}^{n-1} \theta\left(t_{m} q^{j} ; p\right),
$$

or in the additive form:

$$
\left[u_{0}, \ldots, u_{k}\right]_{n} \equiv \prod_{m=0}^{k} \prod_{j=0}^{n-1}\left[u_{m}+j ; \sigma, \tau\right] .
$$

A further generalization of these functions is constructed from $h(n)$ equal to an arbitrary meromorphic Jacobi form in the sense of Eichler and Zagier [5]. The corresponding series were called in [13] as theta hypergeometric series. In this classification, theta hypergeometric series (9) are called balanced if their parameters satisfy the constraint (4).

Elliptic hypergeometric series (9) are called totally elliptic if $h(n)$ is also an elliptic function of all free parameters entering its $\theta_{1}$-functions arguments. It is not possible to have this property if all $u_{i}$ and $v_{i}$ are independent. Therefore such a requirement results in the following additional constraints [13]:

$$
u_{0}+1=u_{1}+v_{1}=\ldots=u_{r}+v_{r}, \quad \text { or } \quad q t_{0}=t_{1} w_{1}=\ldots=t_{r} w_{r} .
$$

These relations are known as the well-poisedness condition for plain and basic hypergeometric series [8]. Thus the total ellipticity concept sheds some light on the origin of the restrictions (10). Totally elliptic hypergeometric series are automatically modular invariant. 
The balancing condition for well-poised series contains a sign ambiguity: $t_{1} \cdots t_{r}$ $= \pm q^{(r+1) / 2} t_{0}^{(r-1) / 2}$. It clearly shows also distinguished character of the parameter $t_{0}$. It is convenient to express $t_{r}$ in terms of other parameters. In this case the function $h(n)$ is invariant with respect to the independent $p$-shifts $t_{0} \rightarrow p^{2} t_{0}$ and $t_{j} \rightarrow p t_{j}, j=1, \ldots, r-1$. However, for odd $r=2 m+1$ we can reach the $t_{0} \rightarrow p t_{0}$ invariance provided we resolve the ambiguity in the balancing condition in favor of the form $t_{1} \cdots t_{2 m+1}=+q^{m+1} t_{0}^{m}$, which is precisely the condition necessary for obtaining nontrivial hypergeometric identities. Thus the notion of total ellipticity (with equal periods) uniquely distinguishes the correct form of the balancing condition.

The elliptic analog of the very-well-poisedness condition consists in adding to (10) of four constraints [13]:

$$
t_{r-3}=t_{0}^{1 / 2} q, \quad t_{r-2}=-t_{0}^{1 / 2} q, \quad t_{r-1}=t_{0}^{1 / 2} q p^{-1 / 2}, \quad t_{r}=-t_{0}^{1 / 2} q p^{1 / 2} .
$$

After simplifications, the very-well-poised theta hypergeometric series ${ }_{r+1} E_{r}$ take the form (no balancing condition is assumed)

$$
{ }_{r+1} E_{r}(\ldots)=\sum_{n=0}^{\infty} \frac{\theta\left(t_{0} q^{2 n} ; p\right)}{\theta\left(t_{0} ; p\right)} \prod_{m=0}^{r-4} \frac{\theta\left(t_{m} ; p ; q\right)_{n}}{\theta\left(q t_{0} / t_{m} ; p ; q\right)_{n}}(-q z)^{n} .
$$

In the limit $p \rightarrow 0$, functions (12) are reduced to the very-well-poised basic hypergeometric series $r-1 \varphi_{r-2}$ of the argument $-q z$ [8]. For even $r$ the balancing condition for (12) has the form $t_{1} \cdots t_{r-4}= \pm q^{(r-7) / 2} t_{0}^{(r-5) / 2}$. For odd $r=2 m+1$, it is appropriate to call (12) balanced if

$$
\prod_{j=1}^{2 m-3} t_{j}=q^{m-3} t_{0}^{m-2}
$$

Then the function $h(n)$ is elliptic in $u_{0}$, i.e. it is invariant with respect to the shift $t_{0} \rightarrow p t_{0}$. Conditions (11) do not spoil this property because the change $t_{0} \rightarrow p t_{0}$ is equivalent (due to the permutational invariance) to a replacement of parameters (11) by $t_{r-3}, p t_{r-2}, p t_{r-1}, t_{r}$ and, so, it does not have an effect upon $h(n)$. The modular invariance (which is not immediately evident due to the dependence of parameters in (11) on the base $p$ ) is preserved due to a similar reasoning.

For the first time, the very-well-poised elliptic hypergeometric series (with a different way of counting the number of parameters and the choice $z=-1$ ) have been considered by Frenkel and Turaev [7]. Their work was inspired by exactly solvable statistical mechanics models built by Date et al [3]. In an independent setting, such functions have been derived by Zhedanov and the author by solving a three term recurrence relation for a self-similar family of biorthogonal rational functions [17.

As shown by Frenkel and Turaev [7, the following summation formula is true:

$$
\begin{aligned}
\sum_{k=0}^{n} \frac{\theta\left(t_{0} q^{2 k} ; p\right)}{\theta\left(t_{0} ; p\right)} & \prod_{m=0}^{5} \frac{\theta\left(t_{m} ; p ; q\right)_{k}}{\theta\left(q t_{0} t_{m}^{-1} ; p ; q\right)_{k}} q^{k} \\
& =\frac{\theta\left(q t_{0} ; p ; q\right)_{n} \prod_{1 \leq r<s \leq 3} \theta\left(q t_{0} / t_{r} t_{s} ; p ; q\right)_{n}}{\theta\left(q t_{0} / t_{1} t_{2} t_{3} ; p ; q\right)_{n} \prod_{r=1}^{3} \theta\left(q t_{0} / t_{r} ; p ; q\right)_{n}}
\end{aligned}
$$

where $\prod_{i=1}^{5} t_{i}=q t_{0}^{2}$ and $t_{4}=q^{-n}, n \in \mathbb{N}$. This means that the special terminating very-well-poised balanced ${ }_{10} E_{9}$ series with $z=-1$ is summable. If we take the 
limit $t_{3} \rightarrow t_{0} / t_{1} t_{2}$, then the termination condition cancels out and (13) becomes a summation formula for an indeterminate ${ }_{8} E_{7}$ series

$$
\sum_{k=0}^{n} \frac{\theta\left(t_{0} q^{2 k} ; p\right)}{\theta\left(t_{0} ; p\right)} \prod_{m=0}^{3} \frac{\theta\left(t_{m} ; p ; q\right)_{k}}{\theta\left(q t_{0} / t_{m} ; p ; q\right)_{k}} q^{k}=\frac{\theta\left(q t_{0}, q t_{1}, q t_{2}, q t_{0} / t_{1} t_{2} ; p ; q\right)_{n}}{\theta\left(q, q t_{0} / t_{1}, q t_{0} / t_{2}, q t_{1} t_{2} ; p ; q\right)_{n}} .
$$

For $p \rightarrow 0$, we obtain an indeterminate summation formula for a very-well-poised balanced ${ }_{6} \varphi_{5}$ basic hypergeometric series. In the additive notation, equality (14) takes the form

$$
\begin{array}{r}
\sum_{k=0}^{n} \frac{\left[u_{0}+2 k\right]}{\left[u_{0}\right]} \frac{\left[u_{0}, u_{1}, u_{2}, u_{0}-u_{1}-u_{2}\right]_{k}}{\left[1,1+u_{0}-u_{1}, 1+u_{0}-u_{2}, 1+u_{1}+u_{2}\right]_{k}} \\
=\frac{\left[1+u_{0}, 1+u_{1}, 1+u_{2}, 1+u_{0}-u_{1}-u_{2}\right]_{n}}{\left[1,1+u_{0}-u_{1}, 1+u_{0}-u_{2}, 1+u_{1}+u_{2}\right]_{n}} .
\end{array}
$$

The general theory of multiple elliptic hypergeometric series is much less developed. Several examples of such series associated with root systems have been investigated by Warnaar [18, van Diejen and the author [4, 13, 15], Rosengren [12, Kajihara and Noumi [9], Rains [11, with the latest contributions coming from the Gustafson's talk at the present conference.

We expect that the approach of 13] to elliptic hypergeometric series is generalizable to multidimensional Riemann theta functions associated with algebraic curves. In this case, we can take as $h(n)$ a meromorphic function upon a compact Riemann surface $S$ of an arbitrary genus $g$. However, the things are much less transparent in this situation. In [15], we started to discuss such generalizations and suggested an extension of sum (15) to a series built from the Riemann theta functions. However, there was a flaw in the formulation of this result. The main purpose of the present note is to lift the Macdonald multiparameter sum described in Theorem 2.27 of [1] to Riemann surfaces of arbitrary genus and to correct a related statement of [15].

The Riemann theta function of $g$ variables $u_{1}, \ldots, u_{g} \in \mathbb{C}$ with characteristics $\alpha=\left(\alpha_{1}, \ldots, \alpha_{g}\right)$ and $\beta=\left(\beta_{1}, \ldots, \beta_{g}\right)$ is defined by the $g$-fold series of the form

$$
\begin{array}{r}
\Theta_{\alpha, \beta}(\mathbf{u} ; \Omega)=\sum_{\mathbf{n} \in \mathbb{Z}^{g}} \exp \left\{\pi i \sum_{j, k=1}^{g}\left(n_{j}+\alpha_{j}\right) \Omega_{j k}\left(n_{k}+\alpha_{k}\right)\right. \\
\left.+2 \pi i \sum_{j=1}^{g}\left(u_{j}+\beta_{j}\right)\left(n_{j}+\alpha_{j}\right)\right\},
\end{array}
$$

where $\Omega_{j k}$ is a symmetric matrix of periods. For general abelian varieties the matrix $\Omega_{j k}$ is arbitrary, and for the Jacobians associated with the Riemann surfaces, it is generated by a basis of holomorphic differentials $\omega=\left(\omega_{1}, \ldots, \omega_{g}\right)$ (see, e.g., [10]). It is evident that for arbitrary $j$ we have

$$
\Theta_{\alpha, \beta}\left(u_{1}, \ldots, u_{j}+1, \ldots, u_{g} ; \Omega\right)=e^{2 \pi i \alpha_{j}} \Theta_{\alpha, \beta}(\mathbf{u} ; \Omega) .
$$

Analogously, we have

$$
\Theta_{\alpha, \beta}\left(u_{1}+\Omega_{1 k}, \ldots, u_{g}+\Omega_{g k} ; \Omega\right)=e^{-\pi i \Omega_{k k}-2 \pi i\left(\beta_{k}+u_{k}\right)} \Theta_{\alpha, \beta}(\mathbf{u} ; \Omega),
$$

where $k=1, \ldots, g$. 
We denote as $\Gamma_{1,2}$ a subgroup of the symplectic modular group $S p(2 g, \mathbb{Z})$ generated by the matrices

$$
\gamma=\left(\begin{array}{ll}
a & b \\
c & d
\end{array}\right) \in S p(2 g, \mathbb{Z})
$$

such that $\operatorname{diag}\left(a^{t} b\right)=\operatorname{diag}\left(c^{t} d\right)=0 \bmod 2$. The action of this group upon the matrix of periods $\Omega$ and the arguments $\mathbf{u}$ of the theta function is defined as

$$
\Omega^{\prime}=(a \Omega+b)(c \Omega+d)^{-1}, \quad \mathbf{u}^{\prime}=\mathbf{u}^{t}(a \Omega+b)^{-1} .
$$

Analogously, we define the tranformed characteristics

$$
\left(\begin{array}{c}
\alpha^{\prime} \\
\beta^{\prime}
\end{array}\right)=\left(\begin{array}{cc}
d & -c \\
-b & a
\end{array}\right)\left(\begin{array}{c}
\alpha \\
\beta
\end{array}\right)+\frac{1}{2}\left(\begin{array}{c}
\operatorname{diag}\left(c^{t} d\right) \\
\operatorname{diag}\left(a^{t} d\right)
\end{array}\right)
$$

Then, the $\Gamma_{1,2}$ group transformation law for theta functions has the form

$$
\Theta_{\alpha^{\prime}, \beta^{\prime}}\left(\mathbf{u}^{\prime} ; \Omega^{\prime}\right)=\zeta \sqrt{\operatorname{det}(c \Omega+d)} e^{\pi i \mathbf{u}^{t}(c \Omega+d)^{-1} c \mathbf{u}_{\Theta}} \Theta_{\alpha, \beta}(\mathbf{u} ; \Omega),
$$

where $\zeta$ is an eighth root of unity [10].

We denote as

$$
v_{j}(a, b) \equiv \int_{a}^{b} \omega_{j}, \quad a, b \in S,
$$

abelian integrals of the first kind. The characteristics $\alpha_{j}, \beta_{j} \in(0,1 / 2)$, such that $4 \sum_{j=1}^{g} \alpha_{j} \beta_{j}=1(\bmod 2)$, are called odd. We denote theta functions with arbitrary (nonsingular) odd characteristics as $[\mathbf{u}], \mathbf{u} \in \mathbb{C}^{g}$, and take the convention that $\left[\mathbf{u}_{1}, \ldots, \mathbf{u}_{k}\right]=\prod_{j=1}^{k}\left[\mathbf{u}_{j}\right]$. For such functions, we have $[-\mathbf{u}]=-[\mathbf{u}]$. It is known that theta functions on Riemann surfaces satisfy the Fay identity [].

$$
\begin{aligned}
& {[\mathbf{u}+\mathbf{v}(a, c), \mathbf{u}+\mathbf{v}(b, d), \mathbf{v}(c, b), \mathbf{v}(a, d)]} \\
& +[\mathbf{u}+\mathbf{v}(b, c), \mathbf{u}+\mathbf{v}(a, d), \mathbf{v}(a, c), \mathbf{v}(b, d)] \\
& =[\mathbf{u}, \mathbf{u}+\mathbf{v}(a, c)+\mathbf{v}(b, d), \mathbf{v}(c, d), \mathbf{v}(a, b)]
\end{aligned}
$$

valid for arbitrary $\mathbf{u} \in \mathbb{C}^{g}$ and $a, b, c, d \in S$ (note that $\mathbf{v}(a, c)+\mathbf{v}(b, d)=\mathbf{v}(b, c)+$ $\mathbf{v}(a, d))$. Here we can replace theta functions independent on the variable $\mathbf{u}$ by prime forms $E(x, y)$ since the cross ratios of these theta functions coincides with the cross ratios of the corresponding prime forms (and therefore they are independent on the choice of odd characteristic for theta functions). However, we find it more convenient to work with one theta function $[\mathbf{u}]$. If we consider the function $[\mathbf{u}+\mathbf{v}(a, b)]$, then, in the appropriate normalization, transformations (17) and (18) correspond to cyclic moves of the point $b$ (or $a$ ) on the Riemann surface along the $A_{i}$ and $B_{i}$ contours, such that $\int_{A_{i}} \omega_{j}=\delta_{i j}$ and $\int_{B_{i}} \omega_{j}=\Omega_{i j}$, respectively [10].

We would like to describe a summation formula for a particular series built from the Riemann theta functions.

Theorem 1. We take a nonnegative integer $n$ and consider $n+1$ arbitrary variables $\mathbf{z}_{k} \in \mathbb{C}^{g}$ and $4 n+4$ different points on a Riemann surface $a_{k}, b_{k}, c_{k}, d_{k} \in S, k=$ $0, \ldots, n$. Then the following multiparameter summation formula for Riemann theta 
functions on algebraic curves takes place

$$
\begin{aligned}
& \sum_{k=0}^{n}\left[\mathbf{z}_{k}\right.\left.+\mathbf{v}\left(b_{k}, c_{k}\right), \mathbf{z}_{k}+\mathbf{v}\left(a_{k}, d_{k}\right), \mathbf{v}\left(a_{k}, c_{k}\right), \mathbf{v}\left(b_{k}, d_{k}\right)\right] \\
& \quad \quad \prod_{j=0}^{k-1}\left[\mathbf{z}_{j}, \mathbf{z}_{j}+\mathbf{v}\left(a_{j}, c_{j}\right)+\mathbf{v}\left(b_{j}, d_{j}\right), \mathbf{v}\left(c_{j}, d_{j}\right), \mathbf{v}\left(a_{j}, b_{j}\right)\right] \\
& \quad \times \prod_{j=k+1}^{n}\left[\mathbf{z}_{j}+\mathbf{v}\left(a_{j}, c_{j}\right), \mathbf{z}_{j}+\mathbf{v}\left(b_{j}, d_{j}\right), \mathbf{v}\left(c_{j}, b_{j}\right), \mathbf{v}\left(a_{j}, d_{j}\right)\right] \\
&=\prod_{k=0}^{n}\left[\mathbf{z}_{k}, \mathbf{z}_{k}+\mathbf{v}\left(a_{k}, c_{k}\right)+\mathbf{v}\left(b_{k}, d_{k}\right), \mathbf{v}\left(c_{k}, d_{k}\right), \mathbf{v}\left(a_{k}, b_{k}\right)\right] \\
& \quad-\prod_{k=0}^{n}\left[\mathbf{z}_{k}+\mathbf{v}\left(a_{k}, c_{k}\right), \mathbf{z}_{k}+\mathbf{v}\left(b_{k}, d_{k}\right), \mathbf{v}\left(c_{k}, b_{k}\right), \mathbf{v}\left(a_{k}, d_{k}\right)\right]
\end{aligned}
$$

Proof. For proving equality (23), we denote as $f_{l}^{(n)}$ and $f_{r}^{(n)}$ its left- and right-hand sides, respectively. We define also

$$
\begin{aligned}
& g_{k}=\left[\mathbf{z}_{k}, \mathbf{z}_{k}+\mathbf{v}\left(a_{k}, c_{k}\right)+\mathbf{v}\left(b_{k}, d_{k}\right), \mathbf{v}\left(c_{k}, d_{k}\right), \mathbf{v}\left(a_{k}, b_{k}\right)\right], \\
& h_{k}=\left[\mathbf{z}_{k}+\mathbf{v}\left(a_{k}, c_{k}\right), \mathbf{z}_{k}+\mathbf{v}\left(b_{k}, d_{k}\right), \mathbf{v}\left(c_{k}, b_{k}\right), \mathbf{v}\left(a_{k}, d_{k}\right)\right],
\end{aligned}
$$

so that $f_{r}^{(n)}=\prod_{k=0}^{n} g_{k}-\prod_{k=0}^{n} h_{k}$.

For $n=0$, equality (23) is reduced to the identity (22). Suppose that (23) is true for $n=0, \ldots, N-1, N \geq 1$. Then we have by induction

$$
\begin{aligned}
& f_{l}^{(N)}=h_{N} f_{l}^{(N-1)} \\
& +\left[\mathbf{z}_{N}+\mathbf{v}\left(b_{N}, c_{N}\right), \mathbf{z}_{N}+\mathbf{v}\left(a_{N}, d_{N}\right), \mathbf{v}\left(a_{N}, c_{N}\right), \mathbf{v}\left(b_{N}, d_{N}\right)\right] \prod_{j=0}^{N-1} g_{j} \\
& \quad=\xi_{N} \prod_{k=0}^{N-1} g_{k}-\prod_{k=0}^{N} h_{k},
\end{aligned}
$$

where

$$
\xi_{N}=h_{N}+\left[\mathbf{z}_{N}+\mathbf{v}\left(b_{N}, c_{N}\right), \mathbf{z}_{N}+\mathbf{v}\left(a_{N}, d_{N}\right), \mathbf{v}\left(a_{N}, c_{N}\right), \mathbf{v}\left(b_{N}, d_{N}\right)\right] .
$$

Using the Fay identity (22), we find that $\xi_{N}=g_{N}$. Therefore, $f_{l}^{(N)}=\prod_{k=0}^{N} g_{k}-$ $\prod_{k=0}^{N} h_{k}=f_{r}^{(N)}$, that is formula (23) is valid for arbitrary $n$.

Remark 1. As noted by the referee, this theorem is a special case of the general construction for telescoping sums:

$$
\sum_{k=0}^{n}\left(x_{k}-y_{k}\right) \prod_{j=0}^{k-1} x_{j} \prod_{j=k+1}^{n} y_{j}=\prod_{j=0}^{n} x_{j}-\prod_{j=0}^{n} y_{j},
$$

whose proof follows similar lines:

$$
\sum_{k=0}^{n}\left(x_{k}-y_{k}\right) \prod_{j=0}^{k-1} x_{j} \prod_{j=k+1}^{n} y_{j}=\sum_{k=0}^{n} \prod_{j=0}^{k} x_{j} \prod_{j=k+1}^{n} y_{j}-\sum_{k=0}^{n} \prod_{j=0}^{k-1} x_{j} \prod_{j=k}^{n} y_{j}
$$

with an obvious cancellation of extra terms in the latter sums. 
For elliptic curves (i.e., for $g=1$ ) formula (23) was proven by Warnaar 18]. Its further degeneration to the trigonometric level leads to a Macdonald identity, which was published for the first time in the Bhatnagar-Milne paper [1] and which generalizes relations obtained by $\mathrm{Chu}$ in [2]. As shown in 11, 18, equalities of such type with special choices of parameters can be used for derivations of more fine structured summation and transformation formulas for bibasic and elliptic hypergeometric series.

We suppose that for some $N>0$ the points $a_{N}, b_{N}, c_{N}, d_{N} \in S$ are such that we hit a zero of a theta function: $\left[\mathbf{v}\left(a_{N}, b_{N}\right)\right]=0$ or $\left[\mathbf{v}\left(c_{N}, d_{N}\right)\right]=0$. Then, using the antisymmetry $\left[\mathbf{v}\left(c_{k}, b_{k}\right)\right]=-\left[\mathbf{v}\left(b_{k}, c_{k}\right)\right]$ equality (23) can be rewritten as

$$
\begin{aligned}
& \sum_{k=0}^{N} \frac{\left[\mathbf{z}_{k}+\mathbf{v}\left(b_{k}, c_{k}\right), \mathbf{z}_{k}+\mathbf{v}\left(a_{k}, d_{k}\right), \mathbf{v}\left(a_{k}, c_{k}\right), \mathbf{v}\left(b_{k}, d_{k}\right)\right]}{\left[\mathbf{z}_{k}+\mathbf{v}\left(a_{k}, c_{k}\right), \mathbf{z}_{k}+\mathbf{v}\left(b_{k}, d_{k}\right), \mathbf{v}\left(b_{k}, c_{k}\right), \mathbf{v}\left(a_{k}, d_{k}\right)\right]} \\
& \quad \times \prod_{j=0}^{k-1} \frac{\left[\mathbf{z}_{j}, \mathbf{z}_{j}+\mathbf{v}\left(a_{j}, c_{j}\right)+\mathbf{v}\left(b_{j}, d_{j}\right), \mathbf{v}\left(c_{j}, d_{j}\right), \mathbf{v}\left(a_{j}, b_{j}\right)\right]}{\left[\mathbf{z}_{j}+\mathbf{v}\left(a_{j}, c_{j}\right), \mathbf{z}_{j}+\mathbf{v}\left(b_{j}, d_{j}\right), \mathbf{v}\left(c_{j}, b_{j}\right), \mathbf{v}\left(a_{j}, d_{j}\right)\right]}=1 .
\end{aligned}
$$

Equivalently, for $\left[\mathbf{v}\left(c_{0}, b_{0}\right)\right]=0$ or $\left[\mathbf{v}\left(a_{0}, d_{0}\right)\right]=0$ we obtain the sum

$$
\begin{gathered}
\sum_{k=0}^{n} \frac{\left[\mathbf{z}_{k}+\mathbf{v}\left(b_{k}, c_{k}\right), \mathbf{z}_{k}+\mathbf{v}\left(a_{k}, d_{k}\right), \mathbf{v}\left(a_{k}, c_{k}\right), \mathbf{v}\left(b_{k}, d_{k}\right)\right]}{\left[\mathbf{z}_{0}, \mathbf{z}_{0}+\mathbf{v}\left(a_{0}, c_{0}\right)+\mathbf{v}\left(b_{0}, d_{0}\right), \mathbf{v}\left(c_{0}, d_{0}\right), \mathbf{v}\left(a_{0}, b_{0}\right)\right]} \\
\quad \times \prod_{j=0}^{k-1} \frac{\left[\mathbf{z}_{j}, \mathbf{z}_{j}+\mathbf{v}\left(a_{j}, c_{j}\right)+\mathbf{v}\left(b_{j}, d_{j}\right), \mathbf{v}\left(c_{j}, d_{j}\right), \mathbf{v}\left(a_{j}, b_{j}\right)\right]}{\left[\mathbf{z}_{j+1}+\mathbf{v}\left(a_{j+1}, c_{j+1}\right), \mathbf{z}_{j+1}+\mathbf{v}\left(b_{j+1}, d_{j+1}\right), \mathbf{v}\left(c_{j+1}, b_{j+1}\right), \mathbf{v}\left(a_{j+1}, d_{j+1}\right)\right]} \\
=\prod_{k=1}^{n} \frac{\left[\mathbf{z}_{k}, \mathbf{z}_{k}+\mathbf{v}\left(a_{k}, c_{k}\right)+\mathbf{v}\left(b_{k}, d_{k}\right), \mathbf{v}\left(c_{k}, d_{k}\right), \mathbf{v}\left(a_{k}, b_{k}\right)\right]}{\left[\mathbf{z}_{k}+\mathbf{v}\left(a_{k}, c_{k}\right), \mathbf{z}_{k}+\mathbf{v}\left(b_{k}, d_{k}\right), \mathbf{v}\left(c_{k}, b_{k}\right), \mathbf{v}\left(a_{k}, d_{k}\right)\right]}
\end{gathered}
$$

In the elliptic case and its further degenerations, such equalities are useful in searching matrices whose inversions are given by simple analytical expressions. It is natural to expect that our relations will lead to a generalization of some of the Warnaar's results on multibasic hypergeometric sums and matrix inversions 18. Moreover, it is worth to analyze possible extensions of the Krattenthaler's determinant from the Warnaar's elliptic case [18 to arbitrary Riemann surfaces.

By special choices of parameters we can try to give to the derived sums hypergeometric type forms. We consider only two particular cases. Substituting

$$
\mathbf{z}_{k}=\mathbf{u}_{0}+\mathbf{v}\left(x_{k}\right), \quad b_{k}=c_{0} \equiv x_{0}, \quad c_{k} \equiv x_{k}, \quad d_{k}=d_{0},
$$

where $\mathbf{u}_{0} \in \mathbb{C}^{g}, \mathbf{v}\left(x_{k}\right) \equiv \mathbf{v}\left(x_{0}, x_{k}\right), k=0,1, \ldots$, and $\mathbf{u}_{2} \equiv \mathbf{v}\left(d_{0}, x_{0}\right), \mathbf{u}_{1}^{(k)} \equiv$ $\mathbf{v}\left(x_{k}, a_{k}\right)$ into (26), we obtain the following identity.

\section{Corollary 2.}

$$
\begin{aligned}
\sum_{k=0}^{n} & \frac{\left[\mathbf{u}_{0}+2 \mathbf{v}\left(x_{k}\right)\right]}{\left[\mathbf{u}_{0}\right]} \frac{\left[\mathbf{u}_{0}-\mathbf{u}_{1}^{(k)}-\mathbf{u}_{2}, \mathbf{u}_{1}^{(k)}\right]}{\left[\mathbf{u}_{0}-\mathbf{u}_{1}^{(0)}-\mathbf{u}_{2}, \mathbf{u}_{1}^{(0)}\right]} \prod_{j=0}^{k-1}\left(\frac{\left[\mathbf{u}_{0}+\mathbf{v}\left(x_{j}\right)\right]}{\left[\mathbf{v}\left(x_{j+1}\right)\right]}\right. \\
& \left.\times \frac{\left[\mathbf{u}_{0}-\mathbf{u}_{1}^{(j)}-\mathbf{u}_{2}+\mathbf{v}\left(x_{j}\right), \mathbf{u}_{1}^{(j)}+\mathbf{v}\left(x_{j}\right), \mathbf{u}_{2}+\mathbf{v}\left(x_{j}\right)\right]}{\left[\mathbf{u}_{1}^{(j+1)}+\mathbf{u}_{2}+\mathbf{v}\left(x_{j+1}\right), \mathbf{u}_{0}-\mathbf{u}_{1}^{(j+1)}+\mathbf{v}\left(x_{j+1}\right), \mathbf{u}_{0}-\mathbf{u}_{2}+\mathbf{v}\left(x_{j+1}\right)\right]}\right) \\
& =\prod_{k=1}^{n} \frac{\left[\mathbf{u}_{0}+\mathbf{v}\left(x_{k}\right), \mathbf{u}_{0}-\mathbf{u}_{1}^{(k)}-\mathbf{u}_{2}+\mathbf{v}\left(x_{k}\right), \mathbf{u}_{1}^{(k)}+\mathbf{v}\left(x_{k}\right), \mathbf{u}_{2}+\mathbf{v}\left(x_{k}\right)\right]}{\left[\mathbf{v}\left(x_{k}\right), \mathbf{u}_{1}^{(k)}+\mathbf{u}_{2}+\mathbf{v}\left(x_{k}\right), \mathbf{u}_{0}-\mathbf{u}_{1}^{(k)}+\mathbf{v}\left(x_{k}\right), \mathbf{u}_{0}-\mathbf{u}_{2}+\mathbf{v}\left(x_{k}\right)\right]}
\end{aligned}
$$


If there would exist a sequence of points $a_{k}$ such that $\mathbf{u}_{1}^{(k)}=\mathbf{u}_{1}^{(0)}$, then four theta functions of the second factor in the left-hand side of (27) would cancel each other and we would obtain an exact $g>1$ analog of the sum (15). Such a condition was assumed implicitly in the derivation of the corresponding formula in 15. However, in general this is possible only for $g=1$ (with the choice $\omega=$ $d u, a_{k}=k+a_{0}, x_{k}=k+x_{0}$ ). As a result, our $g>1$ analog of (15) (which corrects the related formula of [15) does not obey all structural properties of the $g=1$ summation formula. Still, we draw attention to the fact that the right-hand side of (27) satisfies analogs of the balancing and well-poisedness conditions: 1) the sums of arguments of theta functions in the numerator and denominator are equal to each other; 2) the reciprocal theta functions in the numerator and denominator have arguments whose sums are equal to $\mathbf{u}_{0}+2 \mathbf{v}\left(x_{k}\right)$.

Another curious summation formula (inspired by a referee's suggestion) is obtained after substituting $a_{k}=a, c_{k}=b_{0}=c, b_{k+1}=d_{k} \equiv x_{k}$ for $k=0,1, \ldots$, and $\mathbf{z}_{k+1}=\mathbf{z}_{0}+\mathbf{v}\left(c, x_{k}\right)$ for $k>0$ into (26).

\section{Corollary 3.}

$$
\begin{gathered}
\sum_{k=1}^{n} \frac{\left[\mathbf{z}_{\mathbf{0}}, \mathbf{z}_{0}+\mathbf{v}\left(c, x_{k-1}\right)+\mathbf{v}\left(a, x_{k}\right), \mathbf{v}(a, c), \mathbf{v}\left(x_{k-1}, x_{k}\right)\right]}{\left[\mathbf{z}_{0}+\mathbf{v}\left(c, x_{k-1}\right), \mathbf{z}_{0}+\mathbf{v}\left(c, x_{k}\right), \mathbf{v}\left(a, x_{k-1}\right), \mathbf{v}\left(a, x_{k}\right)\right]} \\
=\frac{\left[\mathbf{z}_{0}+\mathbf{v}\left(a, x_{n}\right), \mathbf{v}\left(c, x_{n}\right)\right]}{\left[\mathbf{z}_{0}+\mathbf{v}\left(c, x_{n}\right), \mathbf{v}\left(a, x_{n}\right)\right]}-\frac{\left[\mathbf{z}_{0}+\mathbf{v}\left(a, x_{0}\right), \mathbf{v}\left(c, x_{0}\right)\right]}{\left[\mathbf{z}_{0}+\mathbf{v}\left(c, x_{0}\right), \mathbf{v}\left(a, x_{0}\right)\right]} \\
=\frac{\left[\mathbf{z}_{0}, \mathbf{z}_{0}+\mathbf{v}\left(c, x_{0}\right)+\mathbf{v}\left(a, x_{n}\right), \mathbf{v}(a, c), \mathbf{v}\left(x_{0}, x_{n}\right)\right]}{\left[\mathbf{z}_{0}+\mathbf{v}\left(c, x_{0}\right), \mathbf{z}_{0}+\mathbf{v}\left(c, x_{n}\right), \mathbf{v}\left(a, x_{0}\right), \mathbf{v}\left(a, x_{n}\right)\right]}
\end{gathered}
$$

It is easy also to see that all the derived sums represent "totally abelian" functions, that is they are invariant under arbitrary moves of points on the Riemann surface along the cycles and appropriate $2 g$ shifts of the variables $\mathbf{z}_{k}$ (or $\mathbf{u}_{0}$ ). Analogously, the $S p(2 g, \mathbb{Z})$ modular group invariance is evident by construction (due to the Fay identity properties). As it is clear from our preliminary analysis, theta hypergeometric series for Riemann surfaces with the genus $g>1$ should obey some principally new features with respect to the elliptic case and their determination requires some additional effort.

The author is deeply indebted to the organizers of the Workshop on Jack, HallLittlewood and Macdonald polynomials (Edinburgh, September 23-26, 2003) for invitation to speak there and to the referee for pointing to a flaw in the original formulation of Corollary 2. This work is supported in part by the Russian Foundation for Basic Research (grant No. 03-01-00780). Its final version was prepared

during the author's stay at the Max-Planck-Institut für Mathematik in Bonn whose hospitality is gratefully acknowledged.

\section{REFERENCES}

[1] G. Bhatnagar and S. C. Milne, Generalized bibasic hypergeometric series and their $U(n)$ extensions, Adv. Math. 131 (1997), 188-252.

[2] W. C. Chu, Inversion techniques and combinatorial identities, Bull. Un. Mat. Ital. 7 (1993), 737-760.

[3] E. Date, M. Jimbo, A. Kuniba, T. Miwa, and M. Okado, Exactly solvable SOS models: local height probabilities and theta function identities, Nucl. Phys. B 290 (1987), 231273 . 
[4] J. F. van Diejen and V. P. Spiridonov, Modular hypergeometric residue sums of elliptic Selberg integrals, Lett. Math. Phys. 58 (2001), 223-238.

[5] M. Eichler and D. Zagier, The Theory of Jacobi Forms, Progress in Math. 55, Birkhäuser, Boston, 1985.

[6] J. F. Fay, Theta functions on Riemann surfaces, Lect. Notes in Math. 353, SpringerVerlag, Berlin, 1973.

[7] I. B. Frenkel and V. G. Turaev, Elliptic solutions of the Yang-Baxter equation and modular hypergeometric functions, The Arnold-Gelfand Mathematical Seminars, Birkhäuser, Boston, 1997, pp. 171-204.

[8] G. Gasper and M. Rahman, Basic Hypergeometric Series, Encyclopedia of Mathematics and its Applications 35, Cambridge Univ. Press, Cambridge, 1990.

[9] Y. Kajihara and M. Noumi, Multiple elliptic hypergeometric series. An approach from the Cauchy determinant, Indag. Math. 14 (2003), 395-421.

[10] D. Mumford, Tata Lectures on Theta I, II, Progress in Math. 28, 43, Birkhäuser, Boston, 1983, 1984.

[11] E. M. Rains, Transformations of elliptic hypergeometric integrals, preprint (2003), arXiv:math.QA/0309252

[12] H. Rosengren, Elliptic hypergeometric series on root systems, Adv. Math. 181 (2004), 417-447.

[13] V. P. Spiridonov, Theta hypergeometric series, Asymptotic Combinatorics with Application to Mathematical Physics (St. Petersburg, July 9-22, 2001), Kluwer, 2002, pp. $307-327$.

[14] _ An elliptic incarnation of the Bailey chain, Internat. Math. Res. Notices, no. 37 (2002), 1945-1977.

[15] - Modularity and total ellipticity of some multiple series of hypergeometric type, Theor. Math. Phys. 135 (2003), 836-848.

[16] — Theta hypergeometric integrals, Algebra i Analiz 15 (6) (2003), 161-215 (St. Petersburg Math. J. 15 (6) (2004), 929-967).

[17] V. P. Spiridonov and A. S. Zhedanov, Spectral transformation chains and some new biorthogonal rational functions, Commun. Math. Phys. 210 (2000), 49-83.

[18] S. O. Warnaar, Summation and transformation formulas for elliptic hypergeometric series, Constr. Approx. 18 (2002), 479-502.

[19] E. T. Whittaker and G. N. Watson, A Course of Modern Analysis, Cambridge Univ. Press, Cambridge, 1986

Bogoliubov Laboratory of Theoretical Physics, Joint Institute for Nuclear ReSearch, Dubna, Moscow Region 141980, Russia 